\title{
Carbones de Bajo Rango como Recurso para Enmiendas Húmicas mediante Transformación Microbiana
}

\author{
Nelson O. Valero ${ }^{(1)^{*}}$, José A. Salgado ${ }^{(1)}$, Marlon J. Bastidas ${ }^{(2)}$ \\ (1) Facultad de Ciencias Básicas y Aplicadas, Univ. de La Guajira, Km 5 vía a Maicao, La Guajira, Colombia. \\ (e-mail: nvalerov@uniguajira.edu.co) \\ (2) Facultad de Ingeniería, Universidad de La Guajira, Km 5 vía a Maicao, La Guajira, Colombia.
}

* autor a quien debe ser dirigida la correspondencia.

Recibido Mar. 7, 2018; Aceptado May. 16, 2018; Versión final Jun. 21, 2018, Publicado Oct. 2018

\begin{abstract}
Resumen
El objetivo de este trabajo fue identificar alternativas biotecnológicas para transformar el carbón en compuestos más simples en estado líquido. Se realizó una revisión bibliográfica actualizada relacionada con el uso de la materia orgánica humificada. El análisis documental mostró que la materia orgánica humificada en el suelo mejora las características físicas y químicas del mismo, estimula las poblaciones microbianas y promueve el crecimiento y desarrollo de las plantas. Los carbones de bajo rango se han considerado una buena fuente de sustancias húmicas. La extracción de estas sustancias se puede realizar mediante métodos químicos o a través de la solubilización de carbones de bajo rango utilizando microorganismos. Sin embargo, no ha sido posible el desarrollo de una tecnología eficiente y de bajo costo. En el suelo podría tener lugar un control natural de la transformación del carbón por microorganismos, similar a lo que ocurre con la descomposición de la lignina.
\end{abstract}

Palabras clave: carbones de bajo rango; materia orgánica humificada; biotransformación de carbón; enmiendas húmicas

\section{Low-Rank Coals as a Resource for Humic Amendments through Microbial Transformation}

\begin{abstract}
The aim of this work was to identify biotechnological alternatives for transforming the coal into simpler compounds in liquid state. An updated bibliographic review related to the use of humified organic matter was done. The documentary analysis showed that humified organic matter in the soil improves its physical and chemical properties, stimulates microbial populations and promotes plant growth and development. Lowevolved coals or low rank coals have been considered a good source of humic substances. Extraction of these substances can be performed by chemical methods or through solubilization of low rank coals using microorganisms. However, the development of an efficient and low-cost technology has not been possible. A natural control of coal transformation may take place in the soil in the same way as the decomposition of lignin.
\end{abstract}

Keywords: humic substances, humified organic matter, coal biotransformation 


\section{INTRODUCCIÓN}

El carbón es una roca combustible, sedimentaria, de origen orgánico, está formado principalmente por carbono, hidrógeno, oxígeno, nitrógeno y azufre. El carbón se formó a partir de la vegetación semidescompuesta que se fue consolidando en medio de los estratos de roca y se alteró por los efectos combinados de la presión y el calor durante millones de años (Orem y Finkelman, 2014). El índice de cambio sufrido por un carbón al madurar, desde la turba hasta la antracita, se denomina "rango" y define sus propiedades físicas y químicas finales. Los carbones de bajo rango (CBR) son normalmente blandos y desmenuzables, con un aspecto mate y terroso, presentan niveles de humedad altos, alto contenido de ceniza y material volátil, bajo contenido en carbono fijado y alto contenido de oxígeno; por estas características su potencial energético también es bajo (Giannoulli et al., 2009).

Estructuralmente el carbón es un sistema complejo dominado por una fracción orgánica que puede representar entre el 85 y $95 \%$ de su peso seco. Las fracciones inorgánicas, principalmente aluminosilicatos y piritas comprenden del 5 al 15\%. La fracción orgánica del carbón es compleja y heterogénea, se compone de una matriz que contiene un conjunto de estructuras macromoleculares aromáticas y alifáticas de alto peso molecular, similar a la lignina, el bitumen que consiste en una mezcla heterogénea de moléculas alifáticas y aromáticas de bajo peso molecular (asfaltenos, ceras, resinas) y en tercer lugar están las sustancias húmicas $(\mathrm{SH})$. La organización general de estos componentes se puede describir como un modelo de dos fases: la fase macromolecular, compuesta por una extensa red de macromoléculas, y la fase molecular o móvil, constituida por moléculas de menor tamaño "atrapadas" en función de la polaridad y geometría molecular dentro de la red macromolecular, en conjunto con otras moléculas unidas a la red mediante enlaces no covalentes (Schmiers y Ktipsel, 1997). Una aplicación muy conocida de los CBR es su uso como fuente para la obtención de $\mathrm{SH}$, compuestos de gran importancia por su función en el mantenimiento de algunas propiedades del suelo (Giannoulli et al., 2009). Las SH son utilizadas como enmiendas orgánicas de origen natural, como acondicionadores de suelos y mejoradores de la estructura de los agregados (Piccolo y Mbagwu, 1999); como estimuladores del crecimiento y desarrollo vegetal (Nardi et al., 2002; 2009; Nardi et al., 2016) y como coadyuvantes en procesos de remediación de suelos contaminados (Christanis et al., 2006; Bandeira et al., 2009).

Teniendo en cuenta que el carbón está compuesto por moléculas derivadas de la geoquímica y transformación biológica de tejidos vegetales, la posibilidad de retener agua y aire en los poros de diferente tamaño que conforman su estructura, el contenido de elementos importantes para la nutrición celular tales como nitrógeno, azufre, hierro y algunos elementos traza, además de carbono orgánico relativamente asimilable en la fase móvil, éste material constituye un microhábitat donde es posible la colonización y crecimiento microbiano (Hofrichter y Fakoussa, 2004). Desde comienzos de la década de 1980 se han recopilado numerosas experiencias tendientes a desarrollar procesos de transformación biológica del carbón mediante la solubilización por actividad de hongos y bacterias (Fakoussa, 1981; Fakoussa y Hofrichter, 1999). A esta línea de investigación se ha denominado genéricamente como "transformación microbiana del carbón" o "biotransformación del carbón" (Oboirien et al., 2008).

La biotransformación del carbón es posible porque éste presenta características estructurales semejantes a la lignina, material a partir del cual se originó; por esta razón los microorganismos asociados a la descomposición de la lignina en el suelo también actúan sobre el carbón; sin embargo, existen dificultades para estandarizar y escalar procesos a nivel biotecnológico, debido a que los mecanismos de biotransformación están fuertemente influenciados por condiciones ecológicas propias de los ambientes naturales. La investigación se ha centrado en buscar alternativas biotecnológicas para transformar el carbón en compuestos más simples en estado líquido, fácilmente aprovechables con diferentes propósitos industriales, como combustibles, polímeros y moléculas de valor agregado como materia prima en la industria química (Klein et al., 1999). Si bien un proceso económicamente factible de solubilización de carbón in vitro aún es inviable, el conocimiento generado se puede utilizar para proponer una estrategia de biotransformación de CBR por microorganismos ligninolíticos directamente en el suelo, con el fin de aportar materia orgánica estabilizada que contribuya al mejoramiento de las propiedades de suelos degradados.

\section{MATERIA ORGÁNICA Y ENMIENDAS HÚMICAS EN SUELOS TROPICALES}

La MOS es un conjunto heterogéneo de sustancias conformado en $20 \%$ por moléculas orgánicas simples y de composición definida (carbohidratos, lípidos y proteínas, principalmente), provenientes de la descomposición de restos orgánicos, estas moléculas presentan baja estabilidad y permanencia debido a su rápida mineralización por la microbiota del suelo, por lo cual se denominan como la fracción lábil de la MOS; aproximadamente el $80 \%$ restante está constituido por un conjunto de complejos macromoleculares de composición poco definida, denominados en su conjunto "Materia orgánica humificada" (MOH) o más genéricamente "Sustancias húmicas " $(\mathrm{SH})$, son compuestos polidispersos de alto peso molecular, estables y 
resistentes a la degradación biológica, por lo que reciben la denominación de materia orgánica estabilizada (Nebbioso y Piccolo, 2011; Muscolo et al., 2013).

Químicamente, las SH son agrupaciones supramoleculares complejas originadas en largos procesos de descomposición y repolimerización de los restos orgánicos. Las unidades fundamentales de su macroestructura son compuestos aromáticos de carácter fenólico, unidos por cadenas alifáticas de diferentes tamaños, exhibiendo diferentes grupos funcionales y con cierto contenido de compuestos nitrogenados, tanto cíclicos como alifáticos, sintetizados por ciertos microorganismos del suelo (Piccolo \& Mbagu, 1999; Muscolo et al., 2013). Las SH exhiben naturaleza coloidal y propiedades ácidas, a menudo se encuentran en el suelo asociadas mediante puentes de hidrógeno y fuerzas de Van der Walls a otras fracciones de composición química definida como aminoácidos, azúcares, polisacáridos y proteínas, o a las arcillas, formando complejos arcillo-húmicos, en los cuales radica un sinnúmero de funciones importantes para el suelo (Piccolo, 1996; Theng, 2012). En la actualidad se tienen aproximaciones diferentes a la composición elemental y a la estructura de las SH (Spaccini y Piccolo, 2009); sin embargo, su estudio se dificulta en gran medida por la complejidad intrínseca dado principalmente por la heterogeneidad de procesos y condiciones en las que se originan, debido a esto se ha alcanzado un alto grado de desarrollo en las técnicas utilizadas para su caracterización (Nebbioso y Piccolo, 2011; Muscolo et al., 2013; Drosos et al., 2017). Debido a su dinámica y gran complejidad la MOH podría también denominarse como un "estado de la materia orgánica del suelo".

La mayoría de los suelos agrícolas en regiones tropicales cálidas presentan bajo contenido de materia orgánica, esta tendencia natural se favorece por el clima, que estimula la actividad microbiana induciendo la mineralización de la MOS. Las pérdidas naturales de MOS se aceleran a menudo por condiciones de manejo inadecuado del suelo y una tasa de reposición baja (Piccolo et al., 1997; Liu et al., 2006; Tejada y González, 2007). Frente a esta situación, como medida de compensación se ha generalizado la práctica de adicionar al suelo un grupo variado de productos denominados "enmiendas orgánicas" o "enmiendas húmicas", que por lo general se consideran ricas en materia orgánica estable, esto en complemento a las buenas prácticas de manejo con el fin de mantener o mejorar las propiedades físicas, químicas y biológicas del suelo (Christanis et al., 2006). Este hecho ha generado una dinámica importante en la producción y comercialización de estos insumos orgánicos para la agricultura, mercado dominado por los productos llamados genéricamente "sustancias húmicas"; sin embargo recientemente la atención sobre las SH se ha centrado en un una característica particular, y es su capacidad para incidir sobre el metabolismo vegetal y la arquitectura de sistema radical, por lo cual se considera un elemento fundamental dentro de la tecnología de los fitoestimulantes o bioestimulantes, como estrategia para la intensificación de la agricultura ecológica (Canellas et al., 2015a; Nardi et al., 2017), lo cual resulta fundamental para mejorar la adaptación de las plantas a condiciones limitantes o que generen estrés (Oosten et al., 2017).

\section{USO DE CARBONES POBRES COMO FUENTE DE SH}

La mayoría de enmiendas húmicas se obtienen del compostaje de residuos agrícolas, domésticos, estiércoles, lodos de plantas de tratamiento de aguas residuales, residuos orgánicos municipales, etc.; sin embargo, a menudo la oferta de estos materiales es variable en cantidad y calidad, y esta heterogeneidad dificulta la producción continua y estandarizada de los abonos orgánicos, generando fluctuaciones en costos, oferta disponible y calidad, lo cual limita en cierta medida la adopción de la tecnología por parte de los agricultores (Ribeiro et al., 2000). En complemento a las fuentes tradicionales para la elaboración de enmiendas orgánicas, los carbones de bajo rango o carbones pobres, en algunos países ha sido considerado como una materia prima más homogénea y de oferta relativamente constante (Giannouli et al., 2009; Cubillos et al., 2017; Valero et al., 2018). Desde mediados del siglo XX es común la obtención de SH a partir de leonarditas, carbones pardos, lignitos e incluso carbones bituminosos, de tal suerte que en la actualidad los CBR se han considerado como fuente apropiada para la obtención de enmiendas orgánicas ricas en SH (Chassapis y Roulia, 2008). Se ha concluido que la posibilidad de usar materiales carbonosos como materia prima para el desarrollo de enmiendas y fertilizantes orgánicos para el sector agrícola, depende de las características particulares del material que se pretenda utilizar, puesto que existe una variación significativa entre carbones procedentes de diferentes yacimientos (Taylor y Francis, 2004).

La obtención de SH a partir de carbón generalmente se realiza a través de los mismos métodos básicos de extracción química de $\mathrm{SH}$ a partir del suelo, compost y otros restos de materiales orgánicos estabilizados (Fong et al., 2007; Dick et al., 2017; Pájaro et al., 2017), dentro de los cuales el más común y económico es la extracción con álcali. Como efectos agronómicos de su utilización, se ha demostrado que las SH obtenidas de diferentes clases de carbón desencadenan efectos positivos en la germinación de semillas, contenido y balance nutricional en los tejidos vegetales, absorción de macro y micronutrientes, longitud y crecimiento radical, llenado de frutos y semillas, y calidad en una amplia variedad de plantas de cultivo (Sharif et al., 2002; García et al., 2012; Rose et al., 2014; Olaetxea et al., 2017; Olk et al., 2018). Actualmente se están usando con cierta magnitud los carbones para la extracción de ácidos húmicos $(\mathrm{AH})$ en forma de sales húmicas 
solubles en álcali o AH de carbón ricos en nitrógeno (nitrohumatos de amonio) (Imbufe et al., 2004; 2005; Erro et al., 2016; Lyons \& Genk, 2016). Existen varios procesos patentados para la obtención eficiente de este tipo de sustancias a partir de carbón. También se han hecho investigaciones que determinan la posibilidad de utilizar diferentes tipos de CBR como mejoradores orgánicos y se ha explorado el efecto agronómico de estas aplicaciones (Chassapis et al., 2009; Cubillos et al., 2015).

\section{EFECTOS DE LA MOH EN EL SISTEMA SUELO PLANTA}

Se acepta universalmente la importancia de la materia orgánica del suelo (MOS) como un factor determinante de su salud, calidad y fertilidad, mediante efectos sobre el ciclaje de nutrientes, la retención hídrica y el drenaje, el aumento de la capacidad de intercambio catiónico, la estimulación del crecimiento y desarrollo vegetal, así como la estimulación de la actividad microbiológica, entre otros (Six et al., 2004).

\section{Efectos directos sobre el suelo}

Es aceptado ampliamente que la $\mathrm{MOH}$ puede mejorar directamente algunas propiedades físicas y químicas del suelo, es responsable del aporte y transporte de nutrientes como P, N, Mg y S, entre otros (Imbufe et al., 2005). Las SH favorecen el estado de agregación y en consecuencia inciden en la regulación agua-aire (Piccolo et al., 1997; Piccolo y Mbagwu, 1999); la MOH aumenta la capacidad de intercambio catiónico del suelo, permitiendo un mayor potencial para retener nutrientes y colocarlos a disposición para las plantas, actúa como agente tampón (Varanini y Pinton, 1995); favorece la formación de complejos con $\mathrm{Cu}^{+2} \mathrm{Mn}^{+2}, \mathrm{Zn}^{+2}$ y otros cationes polivalentes, aumentando la disponibilidad de micronutrientes para las plantas (Albuzio et al., 1994), además contribuye al oscurecimiento del suelo e incrementa su capacidad de regulación térmica.

\section{Efectos fisiológicos en las plantas}

Las SH también pueden considerarse bioestimulantes vegetales debido a que exhiben bioactividad sobre las plantas (Barros et al., 2010), este fenómeno conduce a la estimulación del crecimiento, un mejor desempeño en suelos con baja fertilidad o bajo condiciones limitantes y una mejor eficiencia metabólica. La bioactividad de la $\mathrm{MOH}$ comprende una serie de efectos que conducen a respuestas en la arquitectura de la raíz, el metabolismo primario y secundario, y la geometría de las raíces en plantas tratadas con diferentes fracciones húmicas (Canellas y Olivares, 2014). Se sabe que esta actividad en la fracción de ácidos húmicos (AH) genera respuestas semejante a los efectos hormonales de las auxinas, a través de un incremento en la actividad ATPasa $\mathrm{H}^{+}$de la membrana plasmática, induciendo el alargamiento y prolifeación celular (Nardi et al., 2009; Canellas et al., 2015b).

La estimulación de la actividad ATPasa $\mathrm{H}^{+}$también mejora la nutrición vegetal desencadenando un gradiente electroquímico de protones responsable del transporte de iones a través de la membrana celular, por un sistema de transporte secundario (Atiyeh et al., 2002). También se ha demostrado que algunos compuestos húmicos de peso molecular más bajo (principalmente ácidos fúlvicos [AF]) ocasionan cambios en la permeabilidad de la membrana y modulan ciertas actividades enzimáticas por un mecanismo postraduccional indefinido (Nardi et al., 2002; Tomasi et al., 2009) incidiendo en la absorción y transporte de nutrientes. También se ha comprobado que los AH estimulan la germinación y vigor en algunas semillas (Piccolo et al., 1997; Monda et al., 2017). Esto ha desencadenado el uso generalizado de estos compuestos como bioestimulantes, para mejorar la productividad de forrajes, hortalizas, cereales y frutales, entre otros cultivos (Ribeiro et al., 2000; Calvo et al., 2014; Nardi et al., 2016).

\section{Efectos sobre la comunidad microbiana}

La rizodeposición es un importante proceso ecológico a través del cual las plantas suplen con compuestos orgánicos rápidamente asimilables a las poblaciones microbianas que habitan la rizósfera, de esta manera estimulan sus efectos benéficos como el aporte de nitrógeno, fósforo, la protección frente a patógenos y la mitigación del estrés (Ahemad y Kibret, 2014). Se ha comprobado que plantas tratadas con AH exhiben cambios en la producción y diversidad de exudados radicales (Bonkowski, 2004; Calvo et al., 2014). De esta manera, las SH presentan un efecto estimulador indirecto sobre la magnitud y diversidad de la comunidad microbiana de la rizósfera, el cual está mediado por la fisiología de la planta en respuesta a los efectos hormonales de estas sustancias (Puglisi et al., 2009); más aún, se ha demostrado un efecto promotor de las SH sobre la colonización y actividad de bacterias endófitas promotoras del crecimiento vegetal (da Silva Lima et al., 2014; Canellas et al., 2015a), incluso coadyuvando a las plantas a tolerar condiciones de estrés por sequía (da Piedad Melo et al., 2017), y a una mejor asimilación de fosfatos solubles liberados por bacterias solubilizadoras de fosfatos (Giro et al., 2015). 


\section{TRANSFORMACIÓN MICROBIANA DE CARBONES POBRES}

A la fecha se ha comprobado que la presencia de microorganismos en la superficie y el interior del carbón, principalmente el lignito, es un fenómeno natural que contribuye a la transformación y evolución de los minerales y la materia orgánica del mismo, no solo cuando es expuesto a la superficie sino también en el interior de los yacimientos (Bulankina et al., 2007; Yossifova et al., 2011). La mayoría de hongos y bacterias con actividad biotransformadora de CBR son normalmente degradadores de lignina y materiales orgánicos recalcitrantes en el suelo (Filip y Tesarova, 2004); así, las SH presentes en el carbón son liberadas al medio por los microorganismos solubilizadores de carbón, mediante la actividad de enzimas ligninolíticas extracelulares, además de otros mecanismos que incluyen la actividad de enzimas esterasas, agentes quelantes, sustancias surfactantes y sustancias alcalinas (Fakoussa y Hofrichter, 1999). Por la complejidad estructural del carbón, es de esperarse que este sea un proceso lento que no conlleve a una biodegradación completa en corto tiempo, esta liberación lenta de la $\mathrm{MOH}$ presente en el carbón resulta favorable para integrarla al subciclo edáfico del carbono (Senesi et al., 2007). Además, fenómenos como la solubilización de carbón por cometabolismo y la repolimerización de los productos generados en el suelo resultan ventajosos como una forma de controlar el consumo o la degradación completa de los materiales húmicos que contiene (Kidralieva, 2006), puesto que es conveniente que permanezcan en el suelo para poder desencadenar los efectos físicos y químicos sobre el suelo y los efectos fisiológicos benéficos sobre las plantas (Van -Trump et al., 2006).

Existe informaciones que soportan la posibilidad de la aplicación de CBR al suelo como fuente de $\mathrm{SH}$, cuya liberación lenta y sostenida está mediada por la actividad de microorganismos ligninolíticos. En suelos agrícolas, y con mayor efecto en climas semiáridos y áridos, el tratamiento previo con SH derivadas del CBR contribuye a conservar la estabilidad de los agregados cuando el suelo es sometido a la exposición cíclica de humedecimiento y secado (Piccolo et al., 1997); este tratamiento también reduce la erosión causada por la escorrentía (Piccolo et al., 1996). Se ha comprobado que a pH neutro las enzimas peroxidasas en el suelo catalizan la transformación de los AH débilmente enlazados a superestructuras en conformaciones estables de tamaño molecular realmente grande, a través de la formación de enlaces covalentes entre las moléculas húmicas reactivas; también promueven la formación de asociaciones supramoleculares hidrofóbicas de tamaño aparentemente grande. Estos hallazgos sugieren que las peroxidasas del suelo desempeñan un papel importante en la conservación de las SH (Piccolo et al., 2000; Piccolo, 2001; Cozzolino y Piccolo, 2002).

Algunos estudios de caracterización de las sustancias producidas por solubilización microbiana del CBR concluyen que existe alta similaridad en la composición elemental y características de estas sustancias con las SH extraídas con álcali, a partir del mismo CBR y del suelo o materiales compostados (Wilson et al., 1987; Quigley, 1993; Filip y Kubát, 2001). Por ejemplo, Ralph y Catcheside (1996) comprobaron que hongos de la pudrición blanca (Ganoderma applanatum, Merite tremellosus, Perenniporiu tephropora, Phunerochaete chrysosporium, Pycnoporus cinnuburinus, Rigidoporus ulmarius, Trumetes versicolor) y los hongos de la pudrición gris (Gloeophyllum trubeum y Lentinus lepideus) solubilizan carbón en medio líquido, y las sustancias solubles derivadas pueden recuperarse del medio por extracción alcalina, el tratamiento de este producto con $\mathrm{HCl}$ hasta $\mathrm{pH} 2$ separa una fracción insoluble de color marrón que precipita y una fracción soluble de color amarillo, de manera similar a lo que ocurre con las SH (Taylor y Francis, 2004); adicionalmente, mediante análisis FTIR se ha observado que los grupos funcionales presentes en el líquido negro viscoso producido a partir del CBR por el hongo $P$. chrysosporium son similares a los grupos presentes en $\mathrm{AH}$ y AF obtenidos mediante extracción con $\mathrm{NaOH}$ 0,5 M.

Mediante radiomarcaje del lignito en suelos construidos (tecnosoles) en minas de carbón clausuradas se demostró que parte del carbono estabilizado en la materia orgánica del suelo en el horizonte A1 proviene de residuos de carbón (Rumpel y Kogel-Knabner, 2004; Dick et al., 2006). Igualmente, se ha observado que en suelo de minas de carbón rehabilitadas, tras el establecimiento de la comunidad microbiana, el carbón lignito es humificado a razón de 0,25 g. $\mathrm{kg}$ de suelo-1. Año-1 (Rumpel y Kogel-Knabner, 2002). Mediante ensayos de microcosmos, Filip y Tesarova (2004) encontraron que luego de 12 meses la microbiota del suelo utiliza y transforma las $\mathrm{SH}$ de suelos forestales y de praderas en un 63 y $9 \%$, respectivamente. Kwiatkowska et al. (2008) demostraron que en suelos contaminados con carbón éste se integra a la MOS en los horizontes superficiales y conduce al incremento en la relación $\mathrm{C} / \mathrm{N}$.

En suelos recuperados post-minería también se observó una correlación positiva entre la biomasa microbiana y la actividad enzimática del suelo con la presencia de residuos de carbón (Chodak y Niklinska, 2010). Estudios sobre la estabilidad de la enzima manganeso peroxidasa (MnP) en suelos con alto contenido de residuos de carbón, demuestran que esta enzima conserva su actividad en períodos de hasta 4 días después de la aplicación de extractos purificados a partir de cultivos de hongos ligninolíticos, también se observó que aumenta la actividad de MnP nativas tras el incremento del contenido de residuos de carbón en el suelo (Hofrichter et al., 1999). 
Una consideración importante en la evaluación de los materiales derivados del carbón es el grado en que pueden ser potenciales mutágenos o carcinógenos. Se han realizado pruebas para determinar si hidrocarburos aromáticos policíclicos (HAP) o sus análogos heterocíclicos están presentes en los bioextractos resultantes de la solubilización de carbón (Cohen et al., 1991). Además, se han realizado ensayos biológicos directos de actividad mutagénica y análisis estructural de los compuestos; los resultados indican que sólo un pequeño porcentaje del total del material en el bioextracto comprende moléculas suficientemente volátiles y de bajo peso molecular para ser detectadas con éxito por los métodos de análisis. Para comparar el potencial toxicológico de los bioextractos en comparación con la toxicidad de líquidos del carbón producidos termoquímicamente, se han realizado estudios de mutagenicidad microbiana (test de Ames); los resultados no indicaron actividad toxicológica empleando Salmonella typhimurium TA98, lo cual se relaciona con el bajo contenido de PAH detectables instrumentalmente (Cohen et al., 1991). La determinación de elementos traza con valores de 0,$71 ; 2,31 ; 1,73 ; 1,66 ; 0,55 ; 22,43 ; 3,35 ; 2,4 ; 15,11 ; 2,52$ y 0,08 ppm respectivamente para As, $\mathrm{Co}, \mathrm{Pb}, \mathrm{V}, \mathrm{Cu}, \mathrm{Zn}, \mathrm{Ni}, \mathrm{Cr}, \mathrm{B}, \mathrm{Mo}$ y $\mathrm{Cd}$, en muestras de CBR tipo lignito indica que su aplicación al suelo no ofrece riesgo de toxicidad por estos elementos (Valero et al., 2014). Por el contrario, ensayos a nivel de microcosmos con suelos contaminados con DDT revelaron que la adición de CBR tipo lignito e inóculos de bacterias solubilizadoras de carbón conduce a la liberación de la $\mathrm{MOH}$ y reduce la biodisponibilidad de este agroquímico tóxico, tanto por el efecto de las SH liberadas, como por adsorción por parte del CBR (Díaz et al., 2017), así como se ha descrito el papel de la $\mathrm{MOH}$ en procesos de fitorremediación de ambientes contaminados con metales pesados (Bandeira et al., 2009).

Estudios realizados en Colombia desde el año 2009 han revelado que en ambientes influenciados por alto contenido de partículas de carbón, en minas a cielo abierto, se encuentran hongos y bacterias con alta capacidad biotransformadora de carbón (Valero et al., 2009; Duarte et al., 2010; Guevara et al., 2011; Pantoja et al., 2011; Valero et al., 2011). Los AH obtenidos mediante solubilización bacteriana del carbón fueron caracterizados mediante termoquimólisis, resonancia magnética nuclear, espectroscopía infrarroja y cromatografía de filtración en gel, los resultados revelaron una alta similaridad estructural con los AH extraídos con álcali; sin embargo, presentan un carácter menos hidrofóbico de la supraestructura, mayor contenido de grupos funcionales nitrogenados y presencia de algunos ácidos grasos de cadena larga producidos únicamente mediante metabolismo microbiano, este hecho sugiere que las bacterias estudiadas modifican la supraestructura de las SH una vez son solubilizadas a partir del carbón (Valero et al., 2011; Valero et al., 2018). Con respecto a las propiedade bioactivas, se encontró evidencia del efecto fitoestimulador de AH obtenidos mediante la solubilización microbiana del CBR, al tratar plantas de maíz (Pantoja et al., 2016).

Mediante ensayos en invernadero, aplicando CBR entre 1 y $5 \%$ e inóculos de un consorcio microbiano solubilizador de carbón a un material edáfico con bajo contenido de materia orgánica utilizado en la rehabilitación de tierras post minería, se encontró incremento en la respiración, la actividad enzimática y la capacidad de intercambio catiónico del suelo (Valero et al., 2016); por otra parte, la aplicación CBR en polvo o de las SH extraídas ocasionó proliferación de raíces y aumento en la biomasa de plantas de maíz, a la par con una reducción de la densidad aparente del suelo y tendencia al incremento en la estabilidad y el tamaño promedio de los agregados (Valero, 2014). En suelos afectados por salinidad, el tratamiento con lignito inoculado con bacterias solubilizadoras de carbón contribuyó a la recuperación de algunos indicadores relacionados con la agregación del suelo y la actividad biológica (Cubillos et al., 2017).

Teniendo en cuenta la magnitud de las funciones que cumple la $\mathrm{MOH}$ y sus fracciones en el sistema suelo planta, el conjunto de hallazgos descritos anteriormente conlleva a sugerir que cuando se acumulan partículas de carbón en suelos donde existen poblaciones autóctonas de microorganismos ligninolíticos, estas poblaciones pueden biotransformar el carbón e incorporar las $\mathrm{SH}$ presentes en él a la MOH preexistente, tal vez por medio de los mismos mecanismos implicados en el proceso natural de humificación. Considerando las limitantes que se presentan para desarrollar procesos biotecnológicos de biotransformación de carbones residuales a gran escala con el objetivo tradicional de obtener materias primas, combustibles líquidos y compuestos de valor agregado; se puede dirigir la mirada hacia el aprovechamiento de estos carbones mediante su uso en la rehabilitación de suelos afectados por diferentes disturbios, empleando mecanismos de bioaumentación, en caso de ser necesario, con microorganismos nativos biotransformadores de carbón in situ, con el fin de incrementar el contenido húmico del suelo y desencadenar los efectos benéficos de las $\mathrm{SH}$ sobre el suelo, la microbiota benéfica y la planta.

\section{CONCLUSIONES}

La revisión detallada de la información actualizada referente al uso de $\mathrm{MOH}$ como enmienda del suelo permite concluir que: 1. Las propiedades de las SH están definidas por su estructura y las asociaciones que pueden hacer con otras moléculas presentes en el suelo; a su vez, la diversidad y complejidad de sus estructuras derivan de los variados procesos que les dan origen, 2. La aplicación de enmiendas al suelo en forma de $\mathrm{MOH}$ mejoran las propiedades del suelo, promueven la colonización de microorganismos promotores del 
crecimiento vegetal y optimizan el metabolismo de plantas sometidas a condiciones de estrés, lo que permitiría intensificar la agricultura disminuyendo los impactos negativos sobre el ambiente, 3. Los CBR, como subproducto de la minería del carbón, pueden aprovecharse en la obtención de enmiendas orgánicas de óptima calidad y 4. No existen diferencias de gran magnitud entre las $\mathrm{SH}$ producidas por solubilización microbiana y las extraídas químicamente, sin embargo, el metabolismo microbiano genera moléculas con características únicas.

\section{REFERENCIAS}

Ahemad, M. y M. Kibret, Mechanisms and applications of plant growth promoting rhizobacteria: current perspective, Journal of King Saud University-Science, 26(1), 1-20 (2014)

Albuzio, A., G. Concheri, S. Nardi y G. Dell'Agnola, Effect of Humic Fractions of Different Molecular Size on the Development of Oat Seedlings Grown in Varied Nutritional Conditions, Humic Substances in the Global Environment and Implications on Human Health, Senesi, N. y T.M. Miano, pp. 199-204, Elsevier Science BV, Bari, Italia (1994)

Atiyeh, R., S. Lee, C. Edwards, N. Arancon y J. Metzger, The influence of humic acids derived from earthworm-processed organic wastes on plant growth, Bioresource Technology, 84(1), 7-14 (2002)

Bandeira, M., G. Mosca y T. Vamerali, Humic acids affect root characteristics of fodder radish (Raphanus sativus L. var. oleiformis Pers.) in metal-polluted wastes, Desalination, 246(1-3), 78-91 (2009)

Barros, L., L. Pasqualoto y otros siete autores, Bioactivity of Chemically Transformed Humic Matter from Vermicompost on Plant Root Growth, J. Agric. Food Chem., 58 (6), 3681-3688 (2010)

Bonkowski, M., Protozoa and plant growth: the microbial loop in soil revisited, New Phytologist, 162(3), 617-631 (2004)

Bulankina, M., V. Lysak y D. Zvyagintsev, Lignite microorganisms, Biology Bulletin, 34(2), 194-197 (2007)

Calvo, P., L. Nelson y J. Kloepper, Agricultural uses of plant biostimulants, Plant and Soil, 383(1-2), 3-41 (2014)

Canellas, L. y F. Olivares, Physiological responses to humic substances as plant growth promoter, Chemical and Biological Technologies in Agriculture, 1(1), 3 (2014)

Canellas, L. P., S. F. da Silva, D. C. Olk y F.L. Olivares, Foliar application of plant growth-promoting bacteria and humic acid increase maize yields, Journal of Food, Agriculture \& Environment, 13(1), 131-138 (2015a)

Canellas, L., F. Olivares y otros cinco autores, Humic and fulvic acids as biostimulants in horticulture, Scientia Horticulturae, 196, 15-27 (2015b)

Chassapis, K. y M. Roulia, Evaluation of low-rank coals as raw material for Fe and Ca organomineral fertilizer using a new EDXRF method, Int. J. Coal. Geol., 75, 185-188 (2008)

Chassapis, K., M. Roulia y D. Tsirigoti, Chemistry of metal-humic complexes contained in Megalopolis lignite and potential application in modern organomineral fertilization, International Journal of Coal Geology, 78(4), 288-295 (2009)

Chodak, M. y M. Niklińska, Effect of texture and tree species on microbial properties of mine soils, Applied Soil Ecology, 46(2), 268-275 (2010)

Christanis, K., A. Giannouli y otros cuatro autores, Application of soil-improving media produced on lignite-basis on the rehabilitation of post-mining sites, Mineral Wealth, 140, 43-55 (2006)

Cohen, M., B. Wilson y R. Bean, Enzimatic solubilization of coal, Bioprocessing and biotreatment of Coal, Northeastern University, Marcel Dekker Inc, p. 268, Boston, USA (1991)

Cozzolino, A. y A. Piccolo, Polymerization of dissolved humic substances catalyzed by peroxidase. Effects of $\mathrm{pH}$ and humic composition, Organic Geochemistry, 33, 281-294 (2002)

Cubillos, J., N. Valero y A. Peralta, Effect of a low rank coal inoculated with coal solubilizing bacteria for the rehabilitation of a saline-sodic soil in field conditions, Revista Facultad Nacional de Agronomía, 70(3), 8271-8283 (2017)

Cubillos, J., N. Valero y L. Melgarejo, Assessment of a low rank coal inoculated with coal solubilizing bacteria as an organic amendment for a saline-sodic soil, Chemical and Biological Technologies in Agriculture, 2(1), 21 (2015)

da Piedad Melo, A., F. L. Olivares, L. O. Médici, A. Torres-Neto, L. B. Dobbss y L. P, Canellas, Mixed rhizobia and Herbaspirillum seropedicae inoculations with humic acid-like substances improve water-stress recovery in common beans, Chemical and Biological Technologies in Agriculture, 4(1), 6 (2017)

da Silva Lima, L., F. L. Olivares, R. R. de Oliveira, M. R. G. Vega, N. O. Aguiar y L. P. Canellas, Root exudate profiling of maize seedlings inoculated with Herbaspirillum seropedicae and humic acids, Chemical and Biological Technologies in Agriculture, 1(1), 23 (2014)

Díaz-Fuenmayor, K. J., M. Pantoja-Guerra, R. A. Torres-Palma y N. Valero, Changes on the bioavailability of DDT in soil by addition of lignite and coal solubilizing bacteria, Revista Internacional de Contaminación Ambiental, 33(2), 259-268 (2017)

Dick, D., H. Knicker, L. Avila, A. Inda, E. Giasson y C. Bissani, Organic matter in constructed soils from a coal mining area in southern Brazil, Organic geochemistry, 37, 1537-1545 (2006) 
Dick, D., J. Costa, A. Leite y E. Brocchi, Impact of $\mathrm{HNO}_{3}$ solution treatment of South Brazil coal matrices on their chemical composition and humic acids yield, Journal of the Brazilian Chemical Society, 28(5), 790-799 (2017)

Drosos, M., A. Nebbioso, P. Mazzei, G. Vinci, R. Spaccini y A. Piccolo, A molecular zoom into soil Humeome by a direct sequential chemical fractionation of soil, Science of The Total Environment, 586, 807-816 (2017)

Duarte, A., M. Pantoja, E. Guevara y N. Valero, Biosolubilización de lignito (carbón de bajo rango) por hongos del suelo obtenidos en ambientes influenciados por la mina de carbón "El Cerrejón", La Guajira-Colombia, Memorias del I Congreso Iberoamericano de Biotecnología y Biodiversidad, Manizales, Colombia, Septiembre, 8-11 (2010)

Erro, J., O. Urrutia, R. Baigorri, M. Fuentes, A. Zamarreño y J. Garcia, Incorporation of humic-derived active molecules into compound NPK granulated fertilizers: main technical difficulties and potential solutions, Chemical and Biological Technologies in Agriculture, 3(1), 18 (2016)

Fakoussa, R., Coal as a substrate for microorganisms: investigation with microbial conversion of national coals, Tesis de Doctorado, Friedrich-Wilhelms University, Bonn, Germany (1981)

Fakoussa, R.M. y M. Hofrichter, Biotechnology and microbiology of coal degradation, Applied Microbiology and Biotechnology, 521, 25-40 (1999)

Filip, Z. y J. Kubát, Microbial utilization and transformation of humic substances extracted from soils of long-term field experiments, Eur. J. Soil Biol., 37,167-174 (2001)

Filip, Z. y M. Tesarova, MMicrobial degradation and transformation of humic acids from permanent meadow and forest soils, International Biodeterioration \& Biodegradation, 54, 225 - 231 (2004)

Fong, S., L. Seng, N. Majri y H. Mat, A comparative evaluation on the oxidative approaches for extraction of humic acids from low rank coal of Mukah, Sarawak, Journal of the Brazilian Chemical Society, 18(1), 34-40 (2007)

García, J., V. Mora y otros diez autores, Main mechanisms involved in the effects of humic substances on soil-plant systems, Agrociencia Uruguay, 16(3), 188-190 (2012)

Giannoulli, A., K. Stavros y otros seis autores, Evaluation of Greek low-rank coals as potential raw material for the production of soil amendments and organic fertilizers, International Journal of Coal Geology, 77(3-4), 383-393 (2009)

Giro, V. B., K. Jindo y otros cinco autores, Rock phosphate combined with phosphate-solubilizing microorganisms and humic substance for reduction of plant phosphorus demands from single superphosphate, Memorias III International Symposium on Organic Matter Management and Compost Use in Horticulture, 63-68, Murcia - España, 20 al 24 de abril (2015)

Guevara, E., A. Fernández, L. Gómez y N. Valero, Biotransformación de carbón de bajo rango (CBR) tipo lignito por Trichoderma sp. aislado de un lodo carbonoso influenciado por la actividad carbonífera, Memorias del II congreso Iberoamericano de Biotecnología y Biodiversidad, 123-126, Manizales-Colombia, 21 al 24 de septiembre (2011)

Hofrichter, M. y R. Fakoussa, Microbial degradation and modification of coal, Biopolymers I. Lignin, humic substances and coal, Steinbüchel, A. (Ed.), Wiley-VCH, p. 399 - 425, Weinheim, Germany (2004)

Hofrichter, M., D. Ziegenhagen y otros cuatro autores, Degradation of lignite (low-rank coal) by ligninolytic basidiomycetes and their manganese peroxidase system, Appl. Microbiol. Biotechnol., 52, 78-84 (1999)

Imbufe, A., A. Patti, A. Surapaneni, R. Jackson y A. Webb, Effects of brown coal derived materials on pH and electrical conductivity of an acidic vineyard soil, Memorias 3rd Australian New Zealand soils conference, 1-7, Sidney - Australia, 5 al 9 de diciembre (2004)

Imbufe, A., A. Patti, D. Burrow, A. Surapaneni, W. Jackson y A. Milner, Effects of potassium humate on aggregate stability of two soils from Victoria, Australia, Geoderma, 125(3), 321-330 (2005)

Kidralieva, K., Biodegradation of soil humic substances by autochthonous microorganisms, Geophysical Research Abstracts, 8, 3-12 (2006)

Klein, J., D. Catcheside y otros doce autores, Biological processing of fossil fuels, Appl. Microbiol. Biotechnol, 52, 2-15 (1999)

Kwiatkowska, J., M. Provenzano y N. Senesi, Long term effects of a brown coal-based amendment on the properties of soil humic acids, Geoderma, 148(2), 200-205 (2008)

Lázaro, E. y A. Piccolo, Bioactivity of chemically transformed humic matter from vermicompost on plant root growth, Journal. Agric. Food Chem., 58, 3681-3688 (2010)

Liu, X., S. Herbert, A. Hashemi, X. Zhang y G. Ding, Effects of agricultural management on soil organic matter and carbon transformation-a review, Plant Soil and Environment, 52(12), 531 (2006)

Lyons, G. y Y. Genc, Commercial Humates in Agriculture: Real Substance or Smoke and Mirrors?. Agronomy, 6(4), 50 (2016)

Monda, H., V. Cozzolino, G. Vinci, R. Spaccini y A. Piccolo, Molecular characteristics of water-extractable organic matter from different composted biomasses and their effects on seed germination and early growth of maize, Science of the Total Environment, 590, 40-49 (2017)

Muscolo, A., M. Sidari y S. Nardi, Humic substance: relationship between structure and activity, Deeper information suggests univocal findings, Journal of Geochemical Exploration, 129, 57-63 (2013)

Nardi, S., D. Pizzeghello y A. Ertani, Hormone-like activity of the soil organic matter, Applied Soil Ecology, 123, 517-250 (2018) 
Nardi, S., D. Pizzeghello, A. Muscolo y A. Vianello, Physiological effects of humic substances on higher plants, Soil Biol. Biochem, 34 1527-1536 (2002)

Nardi, S., D. Pizzeghello, M. Schiavon y A. Ertani, Plant biostimulants: physiological responses induced by protein hydrolyzed-based products and humic substances in plant metabolism, Scientia Agricola, 73(1), 18-23 (2016)

Nardi, S., P.Carletti, D. Pizzeghello y A. Muscolo, Biological activities of humic substances. Biophysico-chemical processes involving natural nonliving organic matter in environmental systems, 2 (Part 1), http://dx.doi.org/10.1002/9780470494950.ch8, DOI: 10.1002/9780470494950.ch8, John Wiley \& Sons Inc online, 309-335 (2009)

Nebbioso, A. y A. Piccolo, Basis of a humeomics science: chemical fractionation and molecular characterization of humic biosuprastructures, Biomacromolecules, 12(4), 1187-1199 (2011)

Oboirien, B., S. Burton, D. Cowan y S. Hanison, The effect of the particulate phase on coal biosolubilisation mediated by Trichoderma atroviride in a slurry bioreactor, Fuel Processing Technology, 89,123-130 (2008)

Olaetxea, M., D. de Hita y otros diez autores, Hypothetical framework integrating the main mechanisms involved in the promoting action of rhizospheric humic substances on plant root-and shoot-growth, Applied Soil Ecology, 123, $521-537$ (2018)

Olk, D., D. Dinnes, J. Scoresby, C. Callaway y J. Darlington, Humic products in agriculture: potential benefits and research challenges-a review, doi: https://doi.org/10.1007/s11368-018-1916-4, Journal of Soils and Sediments, 1-11 (2018)

Oosten, M.J., O. Pepe, S. Pascale, S. Silletti y A. Maggio, The role of biostimulants and bioeffectors as alleviators of abiotic stress in crop plants, Chemical and Biological Technologies in Agriculture, 4(1), 5 (2017)

Orem, W. y R. Finkelman, Coal Formation and Geochemistry, En Treatise on Geochemistry Second Edition, edited by Heinrich D. Holland and Karl K. Turekian, Elsevier, Oxford, 207-232, ISBN: 9780080983004, https://doi.org/10.1016/B9780-08-095975-7.00708-7 (2014)

Pájaro, A., E. Espinosa, F. Colpas, J. Rodríguez, R. Fernández y E. Meza, Effect of particle size and oxidant concentration in the yield of humic acids from mineral coal using response surface methodology. Revista de la Academia Colombiana de Ciencias Exactas, Físicas y Naturales, 41(160), 361-369 (2017)

Pantoja, M., N. Rodríguez, L. Gómez y N. Valero, Producción de sustancias húmicas mediante solubilización bacteriana de un carbón de bajo rango generado en la mina "El Cerrejón" (La Guajira, Colombia), Memorias del II congreso Iberoamericano de Biotecnología y Biodiversidad, Manizales, Colombia (2011)

Pantoja-Guerra, M., Y. Almanza-Pérez y N. Valero-Valero, Evaluation of auxin-like effect of humic acids on maize through digital image analysis, Revista UDCA Actualidad \& Divulgación Científica, 19(2), 361-369 (2016)

Piccolo, A. y J. Mbagwu, Role of hydrophobic components of soil organic matter in soil aggregate stability, Soil Science Society of America Journal, 63(6), 1801-1810 (1999)

Piccolo, A., A. Cozzolino, P. Conte y R. Spaccini, Polymerization of humic substances by an enzyme-catalyzed oxidative coupling, Naturwissenschaften, 87, 391-394 (2000)

Piccolo, A., G. Pitramellara y J. Mbagwu, Use of humic substances as soil conditioners to increase aggregate stability, Geoderma, 75, 267-277 (1997)

Piccolo, A., Humus and soil conservation, en Humic substances in terrestrial ecosystems, Elsevier science, B.V. 225-264, Amsterdam (1996)

Piccolo, A., The supramolecular structure of humic substances, Soil Science, 166, 810-833 (2001)

Piccolo, A., The supramolecular structure of humic substances: a novel understanding of humus chemistry and implications in soil science, Advances in agronomy, 75, 57-134 (2002)

Puglisi, E., G. Fragoulis y otros 6 autores, Effects of a humic acid and its size-fractions on the bacterial community of soil rhizosphere under maize (Zea mays L.), Chemosphere, 77(6), 80-86 (2009)

Quigley, D., The enzymology and chemistry of coal biosolubilization, Microbial transformations of low ranks coal, Crawford, D. (Ed.), pp. 223, CRC Press, Florida USA (1993)

Ralph, J. y D. Catcheside, Recovery and analysis of solubilised brown coal from cultures of wood-rot fungi, Journal of Microbiological Methods, 27(1), 1-11 (1996)

Ribeiro, H., E. Vasconcelos y J. dos Santos, Fertilisation of potted geranium with a municipal solid waste compost, Bioresource Technology, 73(3), 247-249 (2000)

Rose, M., A. Patti, K. Little, A. Brown, W. Jackson y T. Cavagnaro, A meta-analysis and review of plant-growth response to humic substances: practical implications for agricultura, In Advances in agronomy, Academic Press, 124, 37-89 (2014)

Rumpel, C. y I. Kogel-Knabner, The role of lignite in the carbon cycle of lignite-containing mine soils: evidence from carbon mineralization and humic acid extractions, Organic Geochemistry, 33, 393-399 (2002)

Rumpel, C. y I. Kogel-Knabner, Microbial use of lignite compared to recent plant litter as substrates in reclaimed coal mine soils, Soil. Biol. Biochem., 36, 67-75 (2004)

Schmiers, H. y R. Ktipsel, Macromolecular structure of brown coal in relationship to the degradability by microorganisms, Fuel Processing Technology, 52, 109-114 (1997) 
Senesi, N., C. Plaza, G. Brunetti y A. Polo, A comparative survey of recent results on humic-like fractions in organic amendments and effects on native soil humic substances, Soil biology and biochemistry, 39(6), 1244-1262 (2007)

Sharif, M., R. Khattak y M. Sarir, Effect of different levels of lignitic coal derived humic acid on growth of maize plants, Commun. Soil Sci. Plant Anal., 33, 3567-3580 (2002)

Six, J., H. Bossuyt, S. Degryze y K. Denef, A history of research on the link between (micro) aggregates, soil biota, and soil organic matter dynamics, Soil and Tillage Research, 79(1), 7-31 (2004)

Spaccini, R. y A. Piccolo, Molecular characterization of humic acids stracted of compost at increasing stages of maturity, Soil Biology \& Biochemistry, 41, 1164-1172 (2009)

Taylor, J. y P. Francis, Characterization of humic substances from lignite samples, Energy Sources, 25(1), 23-32 (2004)

Tejada, M. y J. Gonzalez, Influence of organic amendments on soil structure and soil loss under simulated rain, Soil Tillage Res., 93, 197-205 (2007)

Theng, B.K.G, Formation and properties of clay-polymer complexes, Elsevier B.V., Amsterdam-Ntherlands, vol. 4, pp. $501(2012)$

Tomasi, N., T. Kretzschmar y otros 5 autores, Plasma membrane H+-ATPase-dependent citrate exudation from cluster roots of phosphate-deficient white lupin, Plant, cell \& environment, 32(5), 465-475 (2009)

Valero, N., J. Beleño y S. Mancilla, Biotransformación de carbón de bajo rango por bacterias aisladas de microhábitats influenciados por residuos de carbón, Revista Colombiana de Biotecnología, 13(1), 58-65 (2011)

Valero, N., L. Gómez y L. Melgarejo, Supramolecular characterization of humic acids obtained through the bacterial transformation of a low rank coal. J. Braz. Chem. Soc., 1-12 (2018)

Valero, N., L. Gómez, M. Pantoja y R. Ramírez, Production of humic substances through coal-solubilizing bacteria, Brazilian Journal of Microbiology, 45(3), 911-918 (2014)

Valero, N., L. Melgarejo, R. Ramírez, Effect of low-rank coal inoculated with coal solubilizing bacteria on edaphic materials used in post-coal-mining land reclamation: a greenhouse trial, Chemical and Biological Technologies in Agriculture, 3(1), 1-10 (2016)

Valero, N., N. Rodríguez, L. Gómez y L. Diaz, Avances en el estudio de microorganismos nativos para la transformación de carbón de bajo rango para la producción de sustancias húmicas. Memorias del VIII congreso nacional y III internacional de Ciencia y Tecnología del Carbón y Combustibles Alternativos, Bogotá, Colombia (2009)

Valero, N., Transformación microbiana de carbón de bajo rango para inducir cambios en las propiedades del suelo, Tesis Doctoral, Universidad Nacional de Colombia, Facultad de Agronomía, Bogotá D.C. (2014)

Van-Trump, J., I. Sun y J. Coates, Microbial interactions with humic substances, Advances in Applied Microbiology, 60, 55-96 (2006)

Varanini, Z. y R. Pinton, Humic substances and plant nutrition, In Progress in botany, pp. 97-117, Springer, Berlin, Heidelberg (1995)

Wilson, B., R. Bean, J. Franz, B. Thomas, M. Cohen, H. Aronson y E. Gray, Microbial conversion of low rank coal: characterization of biodegraded product, Energy and Fuels, 1, 80-84 (1987)

Yossifova, M., S. Valceva y S. Nikolova, Exogenic microbial activity in coals, Fuel Processing Technology, 92(4), 825-835 (2011) 